\title{
SHORT LINK N ACTS AS A DISEASE MODIFYING OSTEOARTHRITIS DRUG
}

\author{
J. Antoniou ${ }^{1,2}$, L.M. Epure ${ }^{2}$, M.P. Grant ${ }^{2}$, H. Richard ${ }^{3}$, J. Sampalis ${ }^{2}$, P.J. Roughley ${ }^{2} \ddagger$, S. Laverty ${ }^{3}$ \\ and F. Mwale ${ }^{1,2 *}$ \\ ${ }^{1}$ Orthopaedics Research Laboratory, Lady Davis Institute for Medical Research, \\ SMBD-Jewish General Hospital, Montreal, QC, Canada. \\ ${ }^{2}$ Department of Experimental Surgery, Faculty of Medicine, McGill University, Montreal, QC, Canada. \\ ${ }^{3}$ Comparative Orthopedic Research Laboratory, Departement des sciences cliniques, \\ Faculté de Médecine Vétérinaire, Université de Montréal, St. Hyacinthe, QC, Canada. \\ $\ddagger$ This paper is dedicated to the memory of our wonderful friend, \\ Dr. Peter Roughley, who recently passed away.
}

\begin{abstract}
Osteoarthritis $(\mathrm{OA})$ is a degenerative joint disease characterised by a progressive degradation of articular cartilage and underlaying bone and is associated with pain and disability. Currently, there is no medical treatment to reverse or even retard OA. Based on our previous reports, where we establish the repair potential of short Link N (sLN) in the intervertebral disc, a cartilage-like tissue, we hypothesise that sLN may hold similar promises in the repair of articular cartilage. This study aimed to determine if sLN, could prevent OA disease progression.

Skeletally mature New Zealand white rabbits underwent unilateral anterior cruciate ligament transection (ACLT) of their left femorotibial joints to induce joint degeneration typical of OA. Beginning 3 weeks postoperatively, and every three weeks thereafter for 12 weeks, either saline $(1 \mathrm{~mL})$ or sLN (100 $\mu \mathrm{g}$ in $1 \mathrm{~mL}$ saline) was injected intraarticularly into the operated knee. Six additional rabbits underwent sham surgery but without ACLT or post-operative injections. The effects on gross joint morphology and cartilage histologic changes were evaluated.

In the Saline group, prominent erosion of articular cartilage occurred in both femoral condyle compartments and the lateral compartment of the tibial plateau while, sLN treatment reduced the severity of the cartilage damage in these compartments of the knee showing erosion. Furthermore, statistically significant differences were detected between the joint OA score of the saline and sLN treated groups $(p=0.0118)$. Therefore, periodic intraarticular injection of sLN is a promising nonsurgical treatment for preventing or retarding OA progression, by reducing cartilage degradation.
\end{abstract}

Keywords: Osteoarthritis, cartilage repair, tissue engineering, bioactive peptides, short link N.

*Address for correspondence: Fackson Mwale, PhD, FIOR, Orthopaedics Research Laboratory, Lady Davis Institute for Medical Research, SMBD-Jewish General Hospital, Department of Experimental Surgery, Faculty of Medicine, McGill University, 3755 Chemin de la Côte-Sainte-Catherine, Montréal, QC, H3T 1E2, Canada Phone: +1 (514) 3408222 ext 22948...Email: fmwale@jgh.mcgill.ca

Copyright policy: This article is distributed in accordance with Creative Commons Attribution Licence (http://creativecommons.org/licenses/by-sa/4.0/).

\section{Introduction}

Articular cartilage is composed of a dense extracellular matrix (ECM) and a sparse distribution of chondrocytes (Poole et al., 2002; Bayliss and Ali, 1978). Water, collagen and proteoglycans, with other noncollagenous proteins, are present in the ECM. Collagens and proteoglycans are the major macromolecules directly responsible for the viscoelastic load-bearing properties of the articular cartilage. Chondrocytes maintain cartilage ECM by the low-turnover replacement of matrix components. During aging and joint disease, this equilibrium is disrupted and the rate of loss of collagens and proteoglycans from the ECM exceeds the rate of deposition of newly synthesised molecules (Aurich et al., 2006).

Several events trigger joint cartilage destruction, including changes in the mechanical environment, biochemical changes in the ECM, and biological responses of chondrocytes, subchondral bone and synovium to inflammation (Goldring and Otero, 
2011; Roughley, 2001). Osteoarthritis (OA) is a heterogeneous degenerative joint disease in which different causes can result in articular cartilage damage and the destruction of the whole joint. Primary, or idiopathic OA is characterised by a slow progressive degeneration of articular cartilage, increased density or erosion of the subchondral bone, formation of osteophytes, inflammation of the synovium, degeneration of ligaments and the menisci, and hypertrophy of the joint capsule (Conaghan et al., 2015). It is the most common cause of mobility loss, severely affecting the quality of life, work productivity and cost of healthcare, and represents the most prevalent form of musculoskeletal disease worldwide (Elders, 2000; Poole et al., 2002). Other diseases or conditions such as obesity, diabetes, hormone disorders abnormally formed joints at birth (congenital), uric acid crystals in gout, rheumatoid arthritis and other inflammatory conditions of the joints, cause secondary OA increasing mechanical stress on cartilage and repeated trauma to the joint structures (ligaments, bones and cartilage) (Henriksen et al., 2013).

Increased morbidity associated with OA has been shown to contribute indirectly to an increased mortality (Kluzek et al., 2016). However, no drugs are available to modify OA disease progression and current clinical OA management is mainly concerned with symptom reduction, e.g. pain and joint swelling/ stiffness, with oral non-steroidal anti-inflammatory drugs (NSAIDs) as pharmacological treatment at mid-stage of the disease, and arthroplasty, an irreversible procedure, as the final solution to maintain joint function. Timely intervention at early stage in OA progression is critical for preventing or delaying disease development and a key target in preserving proper joint function.

Link N (DHLSDNYTLDHDRAIH), is the 16-amino acid $\mathrm{N}$ terminal region of human link protein, a glycoprotein that stabilises proteoglycan aggregates, which are the major constituents of the extracellular matrix of hyaline cartilage and intervertebral discs (IVDs). It has been shown to increase both collagen and proteoglycan synthesis in chondrocytes (McKenna et al. 1998), in nucleus pulposus cells in vitro, and in ex vivo intact human IVDs (Gawri et al., 2013; Mwale et al., 2003). Furthermore, it was able to increase disc height in a rabbit model of disc degeneration (Mwale et al., 2011). Recent evidence indicates that Link $\mathrm{N}$ activates Smad1/5 signalling in cultured rabbit IVD cells by binding to the bone morphogenetic protein (BMP) type II receptor and upregulating the expression of growth factor BMP-7 (Wang et al., 2013). In addition, Link $N$ was shown to suppress markers of pain in human IVD cells (Noorwali et al., 2018). Link N may therefore provide a therapeutic benefit not only to degenerating discs but also to articular cartilage because of the similar composition of the extracellular matrix. Although Link $\mathrm{N}$ can activate the BMP pathway, it does not stimulate bone formation as has been demonstrated with direct application of BMP growth factors (Antoniou et al., 2012; Wang et al., 2013).

Recently, we found that the first 1-8 residues of Link N (short Link N, sLN) are responsible for its biological activity (Gawri et al., 2014) and that this short peptide can increase proteoglycan synthesis in both types of IVD cells, even in an inflammatory milieu. sLN can stimulate the synthesis of type II collagen and aggrecan in a long-term whole disc organ culture (AlGarni et al., 2016). Recently, it was shown that sLN is as good as Link $\mathrm{N}$ in stimulating proteoglycan content and increasing disc height when administered to the degenerate rabbit disc in vivo at half the molar mass previously shown to be effective with Link N (Mwale et al., 2018). Thus, although sLN appears to be effective at stimulating repair of the IVD in vivo, it remains unknown whether a similar mechanism exists in the repair of other tissues such as cartilage (Antoniou et al., 2012; Liu et al., 1999; Liu et al., 2000; McKenna et al., 1998; Wang et al., 2013).

The purpose of the present study was to determine if $\mathrm{sLN}$ could prevent disease progression in a rabbit model of OA.

\section{Materials and Methods}

\section{Experimental animals}

All animal studies were performed in accordance with the requirements of the Canadian Council on Animal Care. Local ethics committee approval was obtained from the "Comité d'éthique de l'utilisation des animaux (CÉUA)" at the Faculty of Veterinary Medicine, Université de Montréal. Twenty two skeletally mature female (8 months old) New Zealand white rabbits (Charles River, St. Constant, Quebec, Canada) with a mean weight of $3.9 \mathrm{~kg}$ (range: 3.4$5.1 \mathrm{~kg}$ ) were used for this study. Rabbits were housed separately and permitted free cage activity for the duration of the study.

\section{Experimental Design}

A schematic diagram of the study timeline is presented in Fig. 1. Briefly, animals ( $n=22$ rabbits) were randomly divided into 3 groups: 8 rabbits for anterior cruciate ligament transection (ACLT)

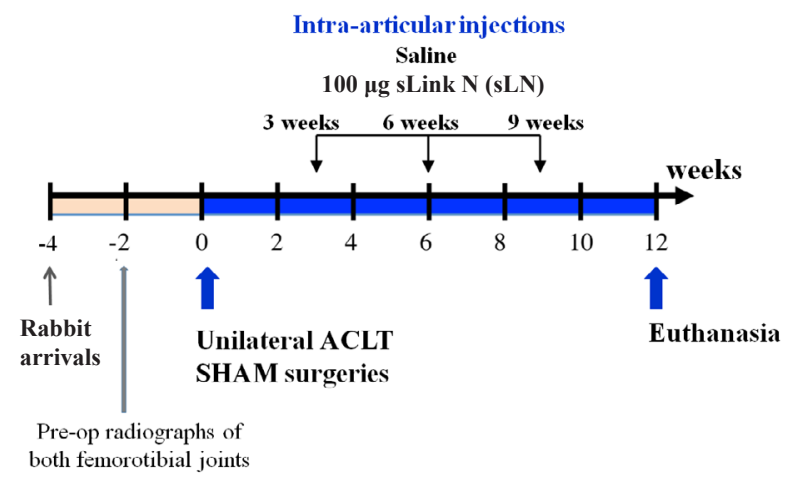

Fig. 1. Schematic presentation of the study timeline. 
followed by sLN treatment, 8 rabbits for ACLT followed by saline treatment, and 6 sham-operated controls without ACLT or any further treatments. Digital radiographs (dorso-plantar and latero-medial views) of all femorotibial joints were performed two weeks prior to surgery, under full sedation, to exclude animals with joint pathology. All unilateral ACLT surgeries were performed at day 0 in 16 rabbits.

Short Link N (sLN) DHLSDNYT (Link N 1-8) with a mass of 964 Da was synthesised by CanPeptide (Pointe Claire, QC, Canada). Treatment was administrated at 3, 6 and 9 weeks post ACLT by intra-articular injections into the operated knee using either $100 \mu \mathrm{g}$ sLN in $1 \mathrm{~mL}$ saline for the sLN group ( $n=8$ rabbits) or $1 \mathrm{~mL}$ saline (vehicle) for the saline group $(n=8)$. All animals were sacrificed 12 weeks post ACLT surgery. The rabbits were weighed before surgery, before each injection and at the end of the study. One of the rabbits assigned to the sLN group showed abnormal behaviour and weight gain before and after surgery (32\% over the average) and was excluded from the study.

\section{ACLT surgeries}

All surgeries were performed by a board-certified veterinary surgeon (specialist) as described previously (Bouchgua et al., 2009). Trimethoprimsulfamide (Tribissen $24 \%$, Schering-Plough Animal Health, Pointe-Claire, QC, Canada), 15 mg/kg, SC, was administered pre-operatively. Rabbits were then pre-medicated, and anaesthesia was induced with xylazine (Novopharm Limited, Animal Health, Toronto, Canada) $5 \mathrm{mg} / \mathrm{kg}$, intramuscular, and ketamine (Bioniche, Animal Health Canada Inc.) $20 \mathrm{mg} / \mathrm{kg}$, intramuscular. Anaesthesia was maintained with 2-2.75\% isoflurane (Baxter Corporation, Mississauga, Canada) via an orotracheal tube, under the supervision of a board-certified veterinary anaesthesiologist. The anterior cruciate ligament (ACL) was transected through a medial parapatellar incision. After ACL transection (ACLT) the skin was subsequently sutured. Analgesia was achieved with the administration of buprenorphine (ScheringPlough Ltd, Welwyn Garden City, UK) $0.03 \mathrm{mg} /$ $\mathrm{kg}$, subcutaneous, pre-operatively and $12 \mathrm{~h}$ later. A fentanyl patch, $25 \mu \mathrm{g} / \mathrm{h}$, was applied to the lumbar region for $72 \mathrm{~h}$ (Duragesic 25, Fentanyl Transdermal System, Janssen-Ortho Inc., Markham, Ontario, Canada) (Bouchgua et al., 2009).

\section{Intraarticular Injections}

Injections were prepared in an aseptic manner, under a biological hood, prior to intra-articular injection. Rabbits were anaesthetised with midazolam $0.2 \mathrm{mg} / \mathrm{kg}$ (Midazolam Sandoz, Sandoz Canada, Boucherville, QC) and dexmetetomidine $0.1 \mathrm{mg} /$ kg mix, intramuscular (Dex-domitor, Zoetis Canada, Kirkland, QC) and Alfaxalone $4 \mathrm{mg} / \mathrm{mL}$, intramuscular, Zoetis Canada (Alfaxan, Jurox, Rutherford, NSW, Australia) $20 \mathrm{~min}$ before injection. The knee was clipped, if there was excess hair, and alcohol was applied over the femoropatellar joint and the limb placed in extension and the central patellar ligament identified. The limb was then placed in slight flexion and the injection was performed under the patella and into the suprapatellar pouch with a $25 \mathrm{G} \times 3 / 4$ " needle. Ten flexions/extensions of the limb were then performed to distribute the treatment evenly within the joint space.

\section{Macroscopic evaluation of joint integrity}

Following euthanasia, the treated femorotibial joints were carefully opened and a map of articular cartilage lesions was performed manually for each joint, after the application of Indian ink to accentuate fibrillation and erosion as described previously (Laverty et al., 2010). Indian ink adheres to fissured cartilage providing contrast with surrounding normal cartilage (Meachim, 1972). The lesions in each compartment (medial femoral condyle [MFC], lateral femoral condyle [LFC], medial tibial plateau [MTP], and lateral tibial plateau [LTP]) were measured and scored by evaluators blinded to the treatment groups according to the criteria outlined in Table 1 (Laverty et al., 2010). Digital images of all the femorotibial articulating surfaces were acquired using a Rebel camera (Nikon, Tokyo, Japan).

\section{Histologic assessment of lesion severity and immunohistochemistry}

The femorotibial joints were harvested and fixed in $10 \%$ neutral-buffered formalin. The specimens were then cut with an ISOMET saw (Buehler, Illinois, USA) with a $15 \mathrm{HC}$ diamond blade $(10.2 \mathrm{~mm} \times 0.3 \mathrm{~mm})$. Sagittal sections were obtained from each femoral condyle and tibial plateau, centred on the most severe lesion. This was performed by visualisation and consultation with corresponding manual maps and digital images. The tissue blocks were decalcified

Table 1. Macroscopic scale for joint integrity.

\begin{tabular}{|l|}
\hline \multicolumn{1}{|c|}{ Scores for macroscopic joint integrity } \\
\hline $1=$ Surface normal in appearance and does not retain Indian ink (Intact surface) \\
\hline $2=$ Fibrillation $<4 \mathrm{~mm}$ \\
\hline $3=4 \mathrm{~mm} \leq$ Fibrillation $<8 \mathrm{~mm}$ \\
\hline $4=$ Fibrillation $\geq 8 \mathrm{~mm}$ \\
\hline $5=$ Erosion $<2 \mathrm{~mm}$ \\
\hline $6=2 \mathrm{~mm} \leq$ Erosion $<5 \mathrm{~mm}$ \\
\hline $7=$ Erosion $\geq 5 \mathrm{~mm}$ \\
\hline
\end{tabular}


for 2 weeks with $14 \%$ ethylenediaminetetraacetic acid solution (Fisher Scientific, Nepean, Ontario), dehydrated through graded alcohols and cleared with toluol prior to being embedded in paraffin wax. $5 \mu \mathrm{m}$ sections were then stained with safranin O-fast green for histological assessment. Multiple sections were also made from normal femoral condyles and tibial plateaus to serve as controls for the assessors. Three independent evaluators, all with prior experience assessing histopathology in this model and including a veterinary American board-certified pathologist, blindly assessed the histological changes employing a previously published scoring system (Table 2) (Laverty et al., 2010).

Sections were deparaffinised and hydrated as previously described (AlGarni et al., 2016). Sections were incubated with BLOXALL (Vector Laboratories, Burlingame, CA) to inactivate the indigenous HRP activity. Sections were blocked and incubated with either anti-BMP 2 polyclonal antibody [1:500] (Abcam, Cambridge, UK, Cat\# ab14933), anti-BMP
7 monoclonal antibody [1:200] (Abcam, Cambridge, UK, Cat\# ab54904) or anti-BMP 4 polyclonal antibody [1:200] (Abcam, Cambridge, UK, Cat\# ab39973) using VECTASTAIN Elite ABC HRP kit (Vector Laboratories, Burlingame, CA) following manufacturer's instructions. Signals were visualised by VECTASTAIN ABC Reagent followed by DAB staining (Vector Laboratories, Burlingame, CA). Sections were counterstained with haematoxylin.

\section{RNA extraction and quantitative real-time PCR}

Three different lots of normal human-knee chondrocytes were purchased from Lonza, Basel, Switzerland. Cells were cultured as micro-pellets at a density of $2.5 \times 10^{5}$ cells/pellet prior to treatment. Pellets were treated for $48 \mathrm{~h}$ with $0.5 \mathrm{~mL}$ medium supplemented with $0.25 \mu \mathrm{g}$ sLN or with vehicle (PBS) alone as a control. Total RNA was extracted using a total RNA mini-kit (Geneaid Biotech Ltd., Taiwan) following manufacturer's instructions. Complementary DNA was synthesised using a

Table 2. Histochemical/histological assessment of articular lesions severity in the ACLT rabbit model of osteoarthritis (Laverty et al., 2010). *observed at one site on section. tobserved at more than one site. t+observed at multiple sites that are in contact.

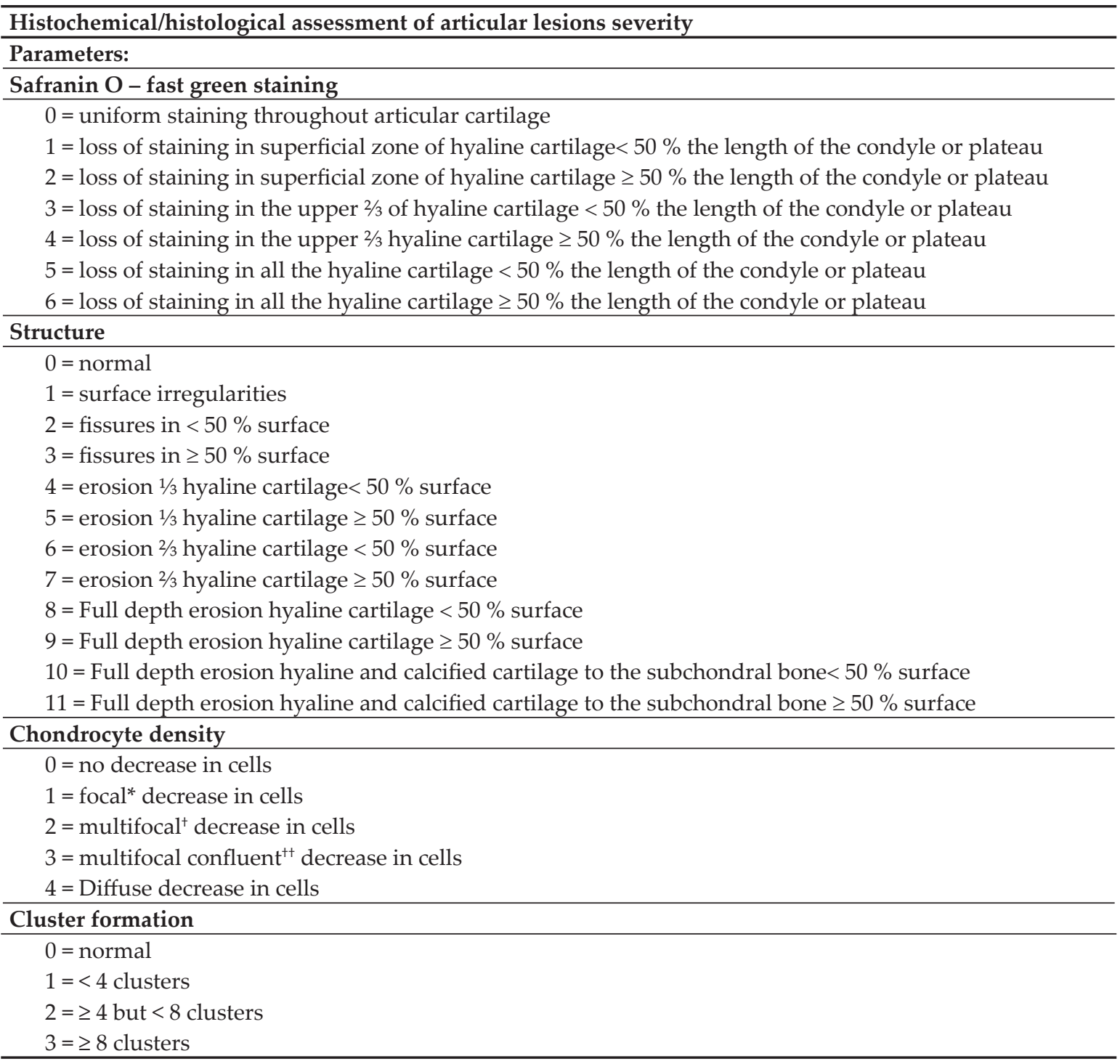


superscript Vilo cDNA synthesis kit (Thermo Fisher Scientific, Waltham, MA). Quantitative real-time PCR of human BMP -2, -4 and -7 was quantified by ABI 7500 fast light cycler using CYBR green master mix (Thermo Fisher Scientific, Waltham, MA) and specific primers (BMP2: Forward- AGTTTATCACCTCAGCAGA; Reverse-CACTTCCACCACGAATCCAT; BMP4: Forward-TAACCTCAGCAGCATCCCT, ReverseTGGTGGGTCCGAGTCTGAT; BMP7: ForwardAGATGCAGCGCGAGATCCTC, ReverseTGGTAGCGTGGGTGGAAGAAT).

Relative mRNA expression level was normalised against GAPDH, as previously described (Antoniou et al., 2012). The one-way analysis of variance (ANOVA) followed by Holm-Sidak's multiple comparisons test was used to assess differences in BMP expression. $p$-values equal to or less than 0.05 were deemed statistically significant.

\section{Statistical analyses}

Macroscopic evaluation of joint integrity

For the categorical variables (scores of the macroscopic evaluation), the macroscopic grades were divided into 2 categories: non-diseased joints with intact surface and diseased joints with evident fibrillation or erosion. A Fisher's exact test was used to compare proportions between sLN and Saline groups.

\section{Histologic assessment of lesion severity}

Categorical variables (the histologic parameters scores at the lesion site) were summarised using frequency distribution per grade. Joint OA score was calculated as the sum of the histologic parameters for each compartment of the knee (safranin O-fast green staining, structure, chondrocyte loss, chondrocyte clusters, tidemark integrity scores). The one-way analysis of variance (ANOVA) followed by HolmSidak's multiple comparisons test was used to assess differences between the sLN, saline and saline groups with respect to OA lesion severity. $p$-values equal to or less than 0.05 were deemed statistically significant. An intra-class coefficient was employed to assess agreement between reviewers for histological assessment (safranin $\mathrm{O}$-fast green and structural scores). Following this independent review, the reviewers met to create a consensus score on samples with a divergence of greater than 2 points.

\section{Results}

\section{Macroscopic joint integrity}

In all joint compartments of the sham group, the articular cartilage surface presented an intact macroscopic appearance with no sign of lesion. In the saline group, fibrillation and prominent erosion occurred in both femoral condyle compartments and the lateral compartment of the tibial plateau (Fig. 2). The macroscopic grade data, categorised as diseased (overt fibrillation and cartilage erosion) or non-diseased, showed $75 \%$ of the saline group with disease in the MFC compartment, compared with $14.3 \%$ in the sLN group $(p=0.041)$. This indicated a statistically significant lower severity of disease for the sLN group compared with the saline group at this site (Table 3).

sLN treatment reduced the severity of the macroscopic cartilage damage in the three compartments of the knee showing erosion. In the LTP and MFC, sLN prevented the erosion, and
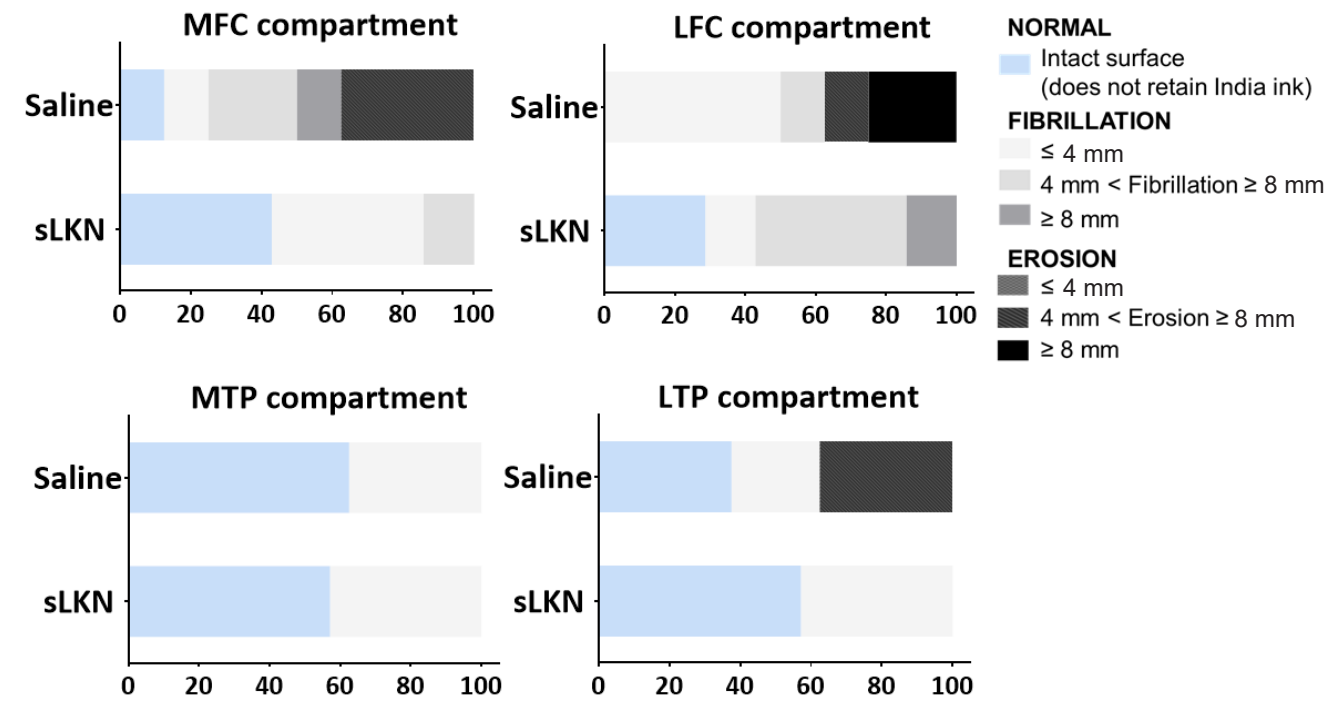

Fig. 2. Macroscopic assessment of the articular surfaces of the knee's compartments. All femorotibial joint compartments in all animals were examined for gross morphologic changes of the articular cartilage and were graded following application of Indian ink; medial femoral condyle (MFC), lateral femoral condyle (LFC), medial tibial plateau (MTP), and lateral tibial plateau (LTP). The 7 grades were divided into 2 categories: diseased joints with evident fibrillation or erosion (scores 3 to 7 ) and non-diseased joints (scores 1 and 2). A Fisher's exact test was used to compare the Saline with the sLN group (percentages of animals with disease in each compartment; Fisher's exact test; ${ }^{*} p=0.041$ ). 
reduceed erosion in the LFC compartment. In the MTP compartment, no erosions occured in either treatment group. There was a significant reduction in the number of erosions on the MFC and a trend in the LTP and LFC compartments. Thus, sLN prevented the severity of the macroscopic damage of the articular cartilage (Fig. 3).

\section{Histologic assessment of the lesion severity Structural parameters}

Histological assessment of safranin-O staining, fissures and erosion of cartilage was performed in order to determine whether sLN has structure modifying properties in the OA joint (Laverty et al., 2010). With respect to histological structural parameter changes, at the end of the study the cartilage in the sham group presented a normal aspect or some fibrillation but never erosions, while partial and full depth erosions were present in 24 (75\%) compartments of the saline group (Table 4). sLN treatment eliminated full depth erosion observed in the saline group. Reduction of lesion severity was observed throughout all the joint compartments with sLN. In the individual compartments, the MFC, LFC and the LTP behaved similarly whereas the MTP showed less severe degradation (no partial erosion) (Table 4) supporting the results of Indian Ink staining.

\section{Proteoglycan assessment}

Safranin-O staining was performed to evaluate proteoglycan loss and replacement. In the sham group, a minor loss of safranin-O staining was observed in the superficial zone of the cartilage in most of the sections, while in the saline group major loss of safranin $\mathrm{O}$ occurred at the lesion site. SLN improved safranin-O staining around the lesions in $14(\sim 30 \%)$ joint compartments, particularly in the medial compartments (Table 4). When individual

Table 3. Proportion of animals with disease (cartilage fibrillation or erosion) on macroscopic evaluation using Indian ink*. The 7 grades were divided into 2 categories for statistical analysis (diseased joints with evident fibrillation or erosion, scores 3 to 7) and non-diseased joints, scores 1 and 2). ${ }^{*}$ Values are percentages of animals with disease in each compartment. Fisher's exact test.

\begin{tabular}{|l|c|c|c|}
\hline \multirow{2}{*}{ Compartment } & \multicolumn{2}{c}{ Group } \\
\cline { 2 - 3 } & $\begin{array}{c}\text { sLN } \\
(n=7)\end{array}$ & $\begin{array}{c}\text { Saline } \\
(n=8)\end{array}$ & $p$ \\
\hline Medial tibial plateau & $0 \%$ & $0 \%$ & 1.000 \\
\hline Lateral tibial plateau & $0 \%$ & $37.5 \%$ & 0.200 \\
\hline Medial femoral condyle & $14.3 \%$ & $75 \%$ & $\mathbf{0 . 0 4 1} \dagger$ \\
\hline Lateral femoral condyle & $57.1 \%$ & $50 \%$ & 1.000 \\
\hline
\end{tabular}
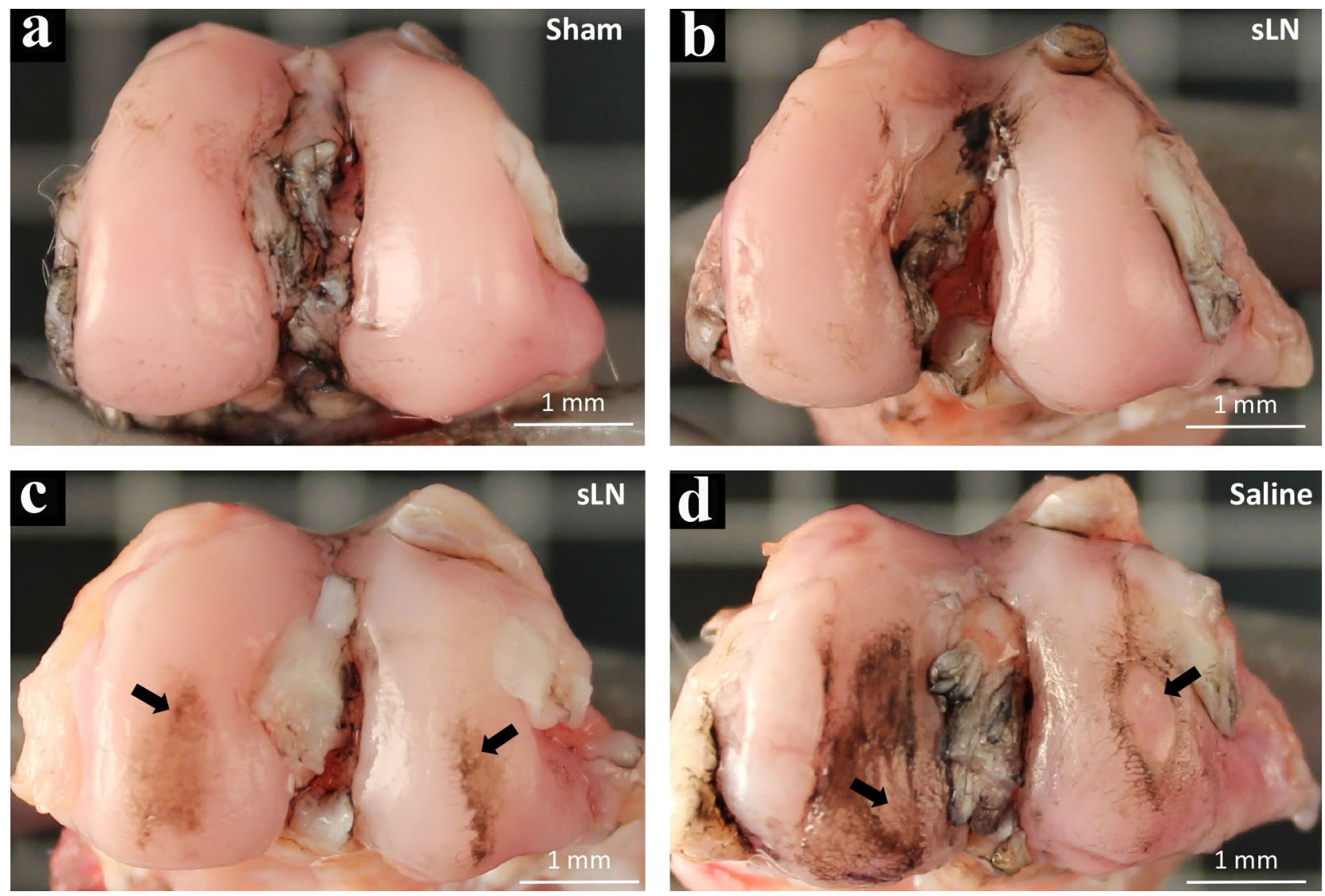

Fig. 3. Representative images of Indian-ink-stained condyles of the knee joints. No uptake of Indian ink indicates an intact surface (score 1); minimal to large focal dark patches of ink uptake indicate mild surface irregularity to overt fibrillation (score 2 to 4); pale area (bone) surrounded by Indian-ink-stained cartilage highlights contiguous to deep erosion in cartilage (score 5 to 7). (a) Sham normal (b) sLink N normal; (c) sLink N fibrillation (black arrows) score 3; and (d) Saline erosion (black arrows) score 6. 


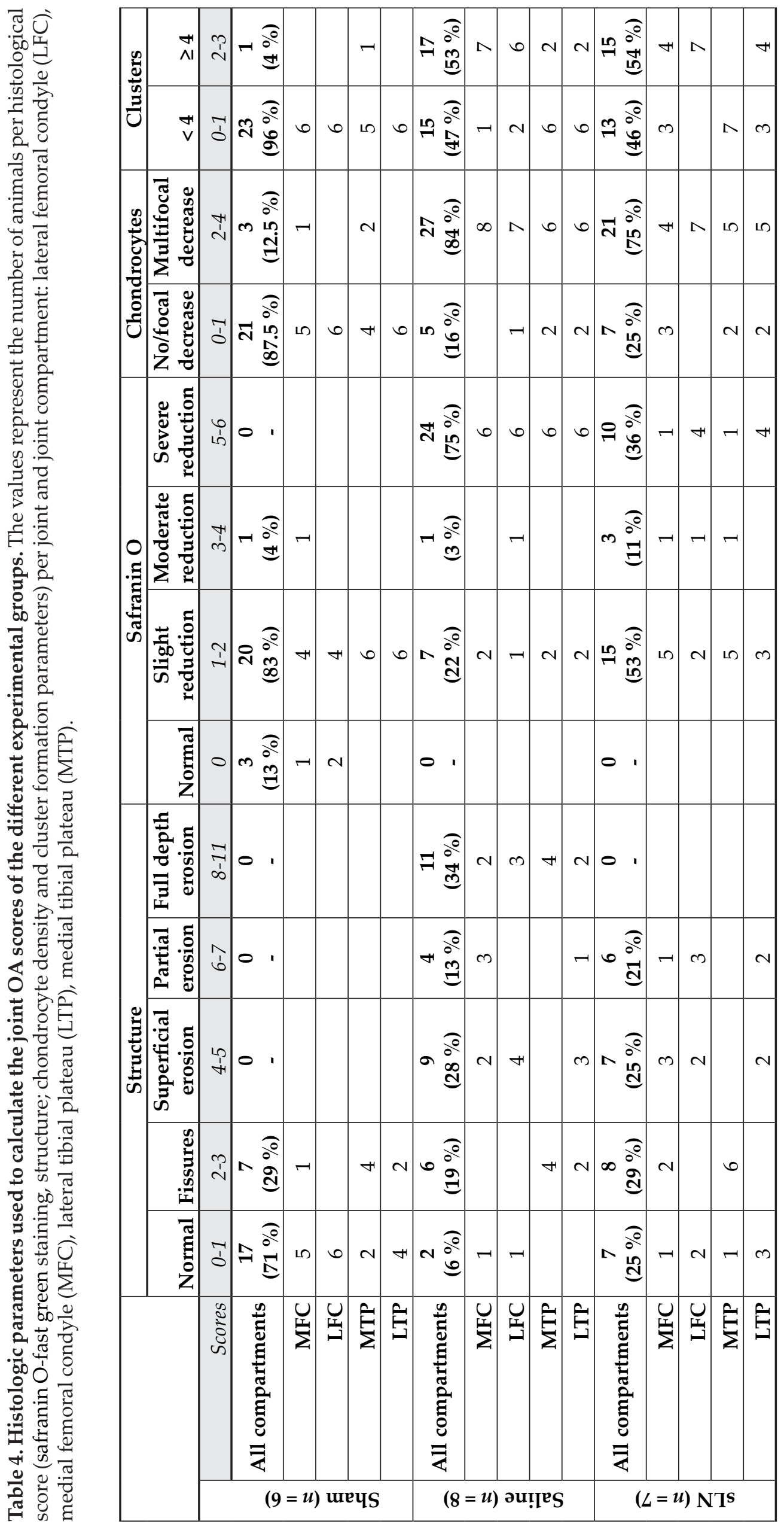


compartments were examined, similar results were observed with preservation of safranin $\mathrm{O}$ by sLN (Table 3).

\section{Cellularity and Cluster assessment}

Cellularity of articular cartilage was assessed to determine whether chondrocyte loss or cluster formation was influenced by sLN (Table 4). In the presence of saline, there was evidence of decreased cell density which appeared to be minimised by the administration of sLink N. Cluster formation was not observed in the sham control, but was a feature of degenerated cartilage of the saline group. sLN had minimal effect on cluster formation (Table 4).

\section{Total joint scores}

OA score decreased significantly $(p=0.0118)$ in the joints of sLN-treated rabbits compared with salinetreated rabbits (Fig. 4). Fig. 5 shows representative images of safranin-O staining patterns in joints from sham, saline and sLN with different degrees of

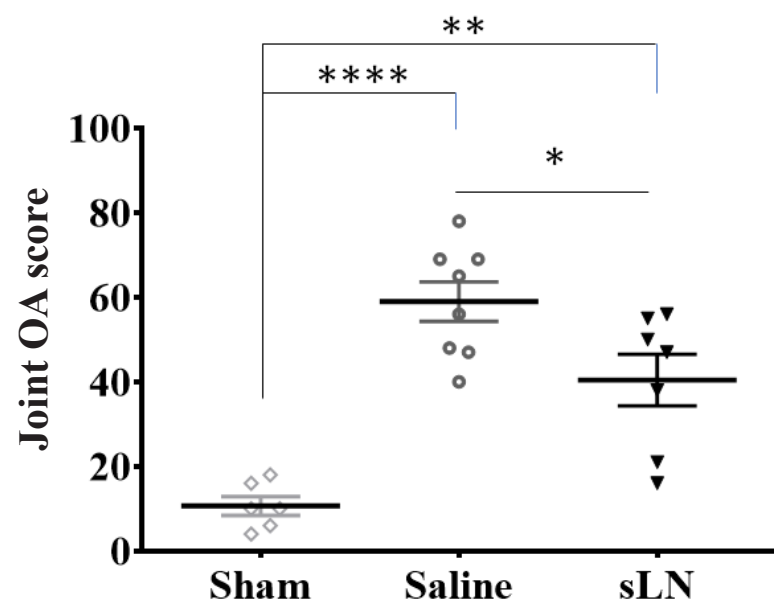

Fig. 4. OA lesion severity of the joints. Scatterplot representation of joint OA histologic scores for sham $(n=6)$, saline $(n=8)$ and $\operatorname{sLN}(n=7)$ groups (Mean \pm SEM; ANOVA, post-hoc Holm-Sidak's multiple comparisons test; ${ }^{*} p=0.0118 ;{ }^{* *} p=0.0011$; **** $p<0.0001)$.
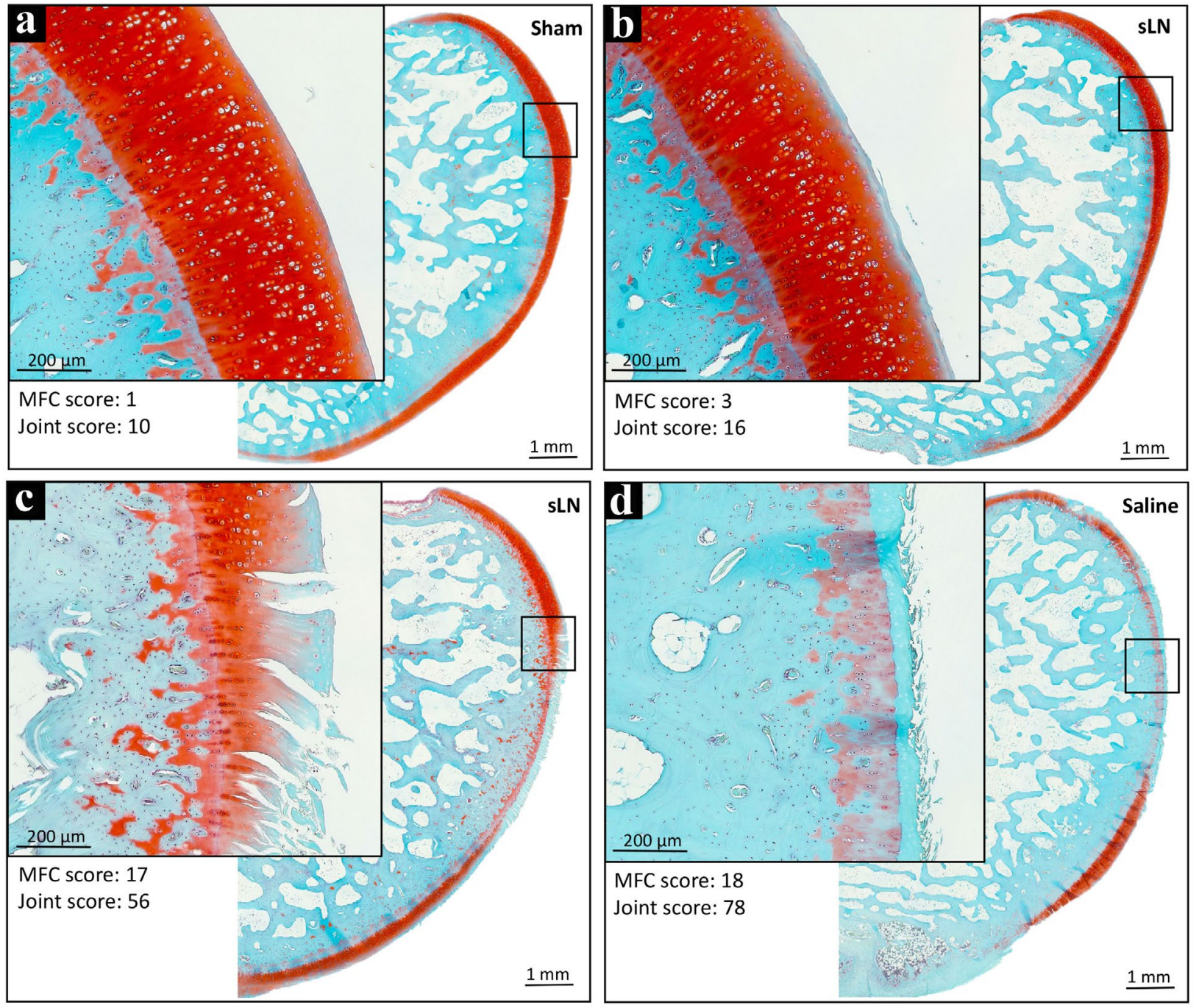

Fig. 5. Representative histopathology of the lesions. Representative safranin O-fast green stained cartilage sections from medial femoral condyles (MFC) presented in Fig. 3, demonstrating scores corresponding to the histochemical/histological grading system (Table 2). The provided images show the complete articular surface of the MFC and a focal image on the surface or lesion: (a) Sham normal joint, OA score 10; (b) sLN normal joint, OA score 21; (c) sLN joint with fibrillation, OA score 56; and (d) Saline joint with erosion; OA score 78. 


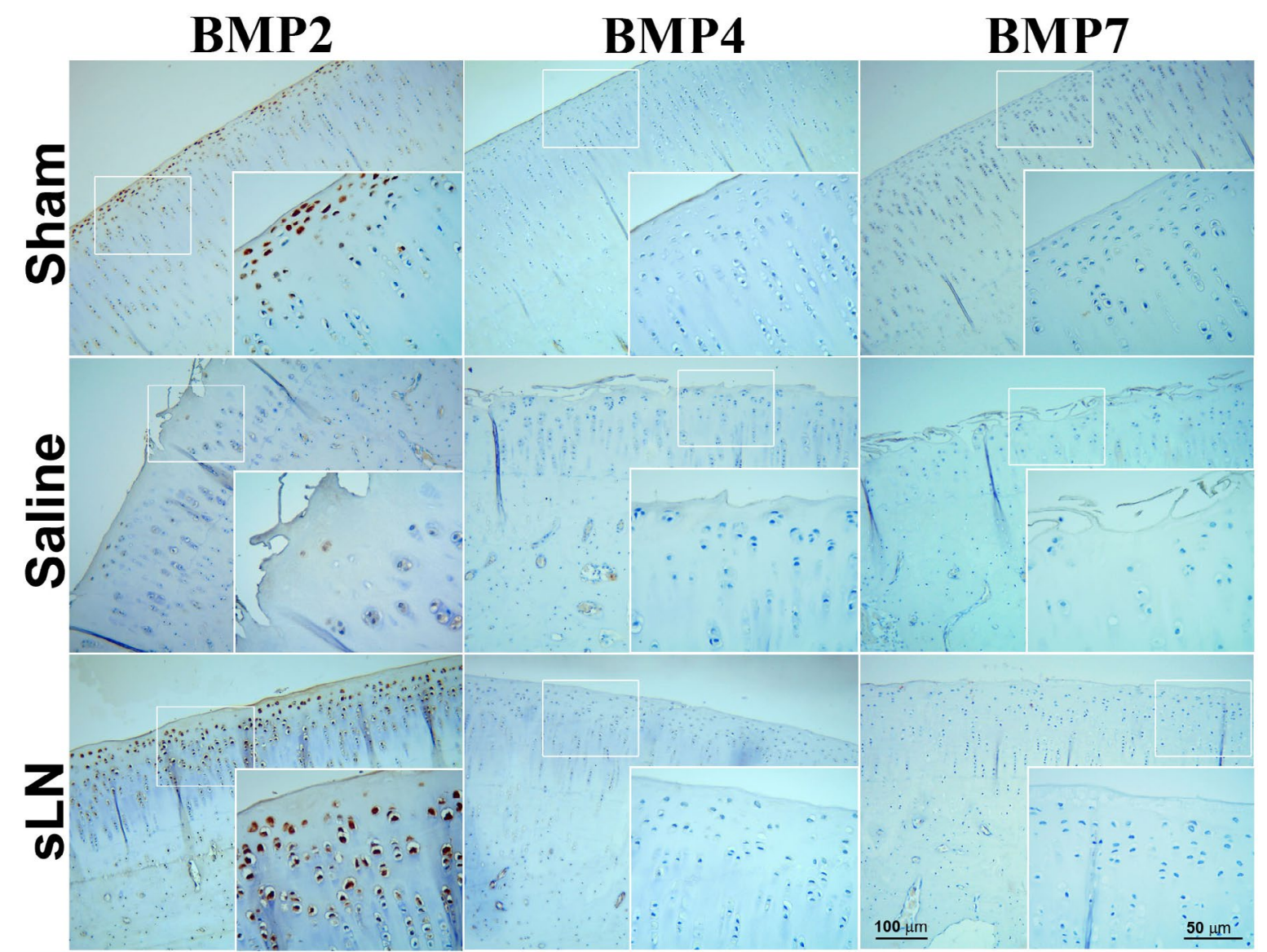

Fig. 6. Immunohistology of BMP expression in rabbit knee joints. Representative BMP-2, -4 , and -7 histological staining of cartilage sections from medial femoral condyles (MFC) presented in Fig. 3 and 5 . The provided images show the partial articular surface surrounding the lesion of the MFC and a focal image on the surface: sham normal joint, OA score 10; saline joint; OA score 78; and sLN; OA score 21. Brown staining demonstrates expression of BMP.

cartilage fibrillation or erosion to show the effect of increase in joint score.

\section{sLN upregulates BMP 2 expression in chondrocytes}

BMP-2 and -7 , have been associated in cartilage repair. In rabbit nucleus pulposus cells, upregulation of BMP-7 and -4 was described as a mechanism for Link N in IVD repair (Wang et al., 2013). To determine whether a similar mechanism exists in our rabbit model of OA, the expression of BMP-2, -4 and -7 were verified in rabbit knee joints treated with sLN compared with sham and saline controls. Although sham animals demonstrated an expression profile for BMP-2, this pattern was lost in saline-treated joints. However, in the sLN treated group, BMP-2 expression was strongly present (Fig. 6). Unlike BMP2, BMP-4 and -7 expression was absent in the joints of all treated animals (Fig. 6).

Previous studies have shown that Link N stimulates both proteoglycan and collagen synthesis (McKenna et al., 1998; Liu et al., 2000) in human articular cartilage. However, a mechanism has not been described. To determine whether sLN induces a similar effect in human chondrocytes on BMP expression as demonstrated in our rabbit model
(Fig. 6), chondrocytes cultured in micro-pellets were treated with sLN for $48 \mathrm{~h}$. Gene expression data indicate that only BMP 2 is significantly upregulated in chondrocytes following sLN treatment (Fig. 7).

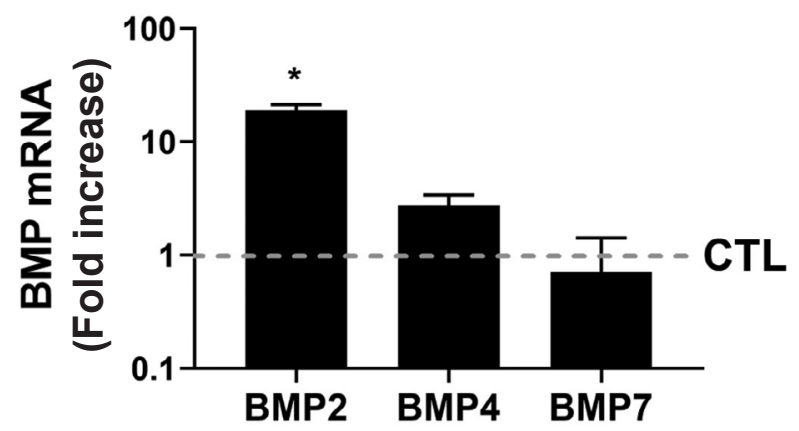

Fig. 7. BMP expression in human chondrocytes following sLN treatment. Human chondrocytes treated for $48 \mathrm{~h}$ with sLN and measured for BMP2, -4 and -7 mRNA expression. Grey hashed line represents control chondrocytes treated with vehicle (saline). Fold increase in expression was taken as a ratio of control. Mean $\pm \mathrm{SD} ; n=3$, ANOVA, post-hoc Holm-Sidak's multiple comparisons test, ${ }^{*} p=0.0158$. 


\section{Discussion}

Previous in vivo studies have established the repair potential of Link $\mathrm{N}$ and sLN in the intervertebral disc, in a rabbit model of disc degeneration (Mwale et al., 2011; Mwale et al., 2018). Several other in vitro studies have demonstrated that Link $\mathrm{N}$ promotes the matrix production in explant cultures of normal human articular cartilage (McKenna et al., 1998; Liu et al., 1999; Liu et al., 2000). In the present study evidence is provided that sLN could prevent OA lesion development or progression in vivo in an ACLT rabbit model of OA. Data indicated that sLN had a protective effect on the severity of cartilage degeneration during the development of $\mathrm{OA}$ that occurred in the LTP, LFC and MFC compartments of the knee, which in the absence of sLN showed prominent erosion. However, sLN had no apparent effect in the MTP compartment. This may be related to the low level of cartilage pathology occurring at this site in the model, as only minor fibrillation was observed in the absence of sLN. It is possible that sLN may only prevent progression of fibrillation to erosion or that exogenous sLN does not affect normal cartilage turnover, but only exerts its influence once matrix degradation exceeds turnover. Although chondrocyte cluster formation was present in both saline and sLN treated animals, only the sLN treated group demonstrated increased proteoglycan content in knee cartilage suggesting a possible chondroprotective effect.

The dosage of sLN was chosen based partly on previous work of intradiscal administration in a rabbit model of disc degeneration (Mwale et al., 2011). Although the chosen dosage of $100 \mu \mathrm{g}$ sLN was shown to be efficacious, the preliminary work on a lower dose $(25 \mu \mathrm{g})$ of intraarticular injection demonstrated no additional improvement (data not shown). To make sure sLN gained access to all the joint surfaces, this was dissolved in $1 \mathrm{~mL}$ saline and the knees were flexed and extended multiple times. It was speculated that repeated knee injections of sLN every 3 weeks would allow for the gradual loss of sLN from the joint space and the amount left would be sufficient to prevent OA progression. Further pharmacokinetic studies will be required to determine the ideal dosage and joint residence time to optimise intra-articular peptide therapy. The experimental duration was selected based on previous studies showing that fibrillation and erosion can occur as early as 4 weeks post ACLT surgery (Spriet et al., 2005) and that 12 weeks is sufficient for lesions to develop with full depth cartilage erosion (Bouchgua et al., 2009; Laverty et al., 2010; Tiraloche et al., 2005).

BMP signalling plays an integral role in skeletal development and bone formation (Salazar et al., 2016). Evidence suggests that BMPs are also important in articular cartilage homeostasis. For instance, gradual wearing of articular cartilage was observed with age in mice with a conditional knockout to BMP-receptor type 1A (BMPR-1A), resembling the process of OA in humans (Rountree et al., 2004). In addition, BMPR1A expression corelated with the progression of OA in cartilage lesions of human knee joints. Evidence in patients with circumscribed cartilage lesions implicate BMP-2 as an important factor in cartilage repair as a statistically significant correlation was observed with improvement of functional knee scores following regenerating surgery (Schmal et al., 2010).

In another study, upregulation of BMP-2 and increased BMP-2 signalling was also found following mechanical stress in cartilage explants (Dell'Accio et al., 2006). In addition to mechanical stress, proinflammatory cytokines, interleukin (IL)- $1 \beta$ and tumour necrosis factor (TNF)- $\alpha$ can stimulate the expression of BMP-2 in chondrocytes suggesting localised inflammation can induce an anabolic response (Fukui et al., 2003).

In addition to BMP-2, BMP-7 (osteogenic protein-1) has demonstrated an important role in articular cartilage homeostasis and repair (Stove et al., 2006; Sekiya et al., 2009; Hayashi et al., 2008; Badlani et al., 2008). Delivery of BMP-7, by an osmotic pump to the joint, has been studied both in a prophylactic (Badlani et al., 2008) or therapeutic protocol (Rountree et al., 2004). In the first study, administration of recombinant human BMP-7 at the same time as the ACLT led to decreased OA severity at a gross and histological level (Rountree et al., 2004). At a microscopic level, BMP-7 treatment considerably decreased the amount of articular cartilage loss measured histologically. BMP-7 treatment 4 weeks after the ACLT to allow for formation of arthritis before treatment, although less effective, showed its potential to reduce degradation of articular cartilage in OA. Others, found that weekly intra-articular injection of BMP-7 was able to inhibit progression of OA in rabbits with ACLT (Hayashi et al., 2008). In vitro studies have also demonstrated that BMP-7 can stimulate proteoglycan synthesis in human OA chondrocytes (Stove et al., 2006).

Our data indicated that sLN upregulates BMP-2 in human chondrocytes and throughout the lesion in our rabbit model of OA. Surprisingly unlike previous reports, where Link $\mathrm{N}$ was shown to induce disc repair through upregulation of BMP-7 and to a lesser extent of BMP-4 by interaction with the BMP type II receptor (Wang et al., 2013), the current study suggested that the anabolic activity of sLN in cartilage may be operating through stimulation of BMP-2 expression.

Although the rabbit ACLT model is widely used to test disease-modifying OA drugs (DMOAD), it has its limitations. Clinical OA typically manifest over the course of several years not rapidly over a few weeks, as is the case in our ACLT model. Joint instability generated by ACLT provides a challenge for testing structure/DMOADs, as the instability induced by ACLT that caused the OA is maintained post-surgery and during treatment. This may arguably provide a greater hurdle for DMOAD 
discovery. The fact that the MFC is the site with the greatest loading (Yoshioka et al., 1996) and the most severely affected compartment in our ACLT model makes the beneficial effect of sLN even more remarkable. Thus, pharmacologically, sLN therapy could be a novel therapeutic approach for treating OA by preventing disease progression. Since ACLT affects mostly cartilage it would be beneficial to study sLN in different OA models to investigate changes on other tissues such as cortical bone and synovium since OA is a disease of the whole joint.

Currently, there is no therapy specifically targeting cell death in human OA. However, preclinical studies in animal models suggest that inhibition of caspases might be a viable option to slow OA progression (Del Carlo et al., 2008). Intraarticular administration of sLN may have a chondroprotective effect, enhancing the synthesis of new cartilage matrix, in addition to preventing further matrix degradation. A second major advantage of sLN over a growth factor such as BMP-2 and BMP-7 for therapeutic use is its straightforward chemical synthesis versus the complex expression and purification of a recombinant protein.

In conclusion, the results suggest that intraarticular injection of sLN is a promising nonsurgical treatment for early symptomatic stages of OA progression. One of the holy grails in rheumatology has been the search for a DMOAD. Although there have been advances in our understanding of $\mathrm{OA}$ and the development of new agents and treatment strategies, there are no DMOAD on the market (Hunter, 2011). Based on the findings of the current work, sLN represents one example that can be useful as a therapeutic agent with the features of a DMOAD.

\section{Acknowledgements}

This work was supported by the Canadian Institutes of Health Research, the Natural Sciences and Engineering Research Council of Canada (NSERC) and the Quebec Network for Cell and Tissue Therapies. We also thank Allan Mandelzys for his helpful comments on the manuscript.

Competing Interests: Two of the authors (JA and FM) are co-founders of Trepso Therapeutics Inc. that owns the patent for use of sLink N, entitled "Methods and Compositions for Treatment of Cartilage and Disc Disorders", as described in the PCT Patent Application No. PCT/CA2014/000656, filed on 4 April 2014. FM, JA, PJR, LME and MPG are the inventors of sLink $\mathrm{N}$. The remaining authors have no competing interests to declare.

Authors' Contributions: FM, SL, JA conceptualised the experiments and supervised the work; SL and HR performed the in vivo experiments, processed the samples and evaluated the tissue samples. MPG performed the in vitro experiments. HR, LME and MPG collected the data; JS and LME performed the statistical analysis. FM, PJR, SL and LME prepared the manuscript; LME, MPG and HR prepared the figures. All authors approved the final version of the manuscript for publication.

\section{References}

AlGarni N, Grant MP, Epure LM, Salem O, Bokhari R, Antoniou J, Mwale F (2016) Short link N stimulates intervertebral disc repair in a novel longterm organ culture model that includes the bony vertebrae. Tissue Eng Part A 22: 1252-1257.

Antoniou J, Wang HT, Alaseem AM, Haglund L, Roughley PJ, Mwale F (2012) The effect of Link N on differentiation of human bone marrow-derived mesenchymal stem cells. Arthritis Res Ther 14: R267.

Aurich M, Mwale F, Reiner A, Mollenhauer JA, Anders JO, Fuhrmann RA, Kuettner KE, Poole AR, Cole AA (2006) Collagen and proteoglycan turnover in focally damaged human ankle cartilage: evidence for a generalized response and active matrix remodeling across the entire joint surface. Arthritis Rheum 54: 244-52.

Badlani N, Inoue A, Healey R, Coutts R, Amiel D (2008) The protective effect of OP-1 on articular cartilage in the development of osteoarthritis. Osteoarthritis Cartilage 16: 600-606.

Bayliss MT and Ali SY (1978). Age-related changes in the composition and structure of human articularcartilage proteoglycans. Biochem J 176: 683-693.

Bouchgua M, Alexander K, d'Anjou MA, Girard CA, Carmel EN, Beauchamp G, Richard H, Laverty S (2009) Use of routine clinical multimodality imaging in a rabbit model of osteoarthritis - part I. Osteoarthritis Cartilage 17: 188-196.

Conaghan PG, Porcheret M, Kingsbury SR, Gammon A, Soni A, Hurley M, Rayman MP, Barlow J, Hull RG, Cumming J, Llewelyn K, Moscogiuri F, Lyons J, Birrell F (2015) Impact and therapy of osteoarthritis: the Arthritis Care OA Nation 2012 survey. Clin Rheumatol 34: 1581-1588.

Del Carlo M Jr and Loeser RF (2008) Cell death in osteoarthritis. Curr Rheumatol Rep 10: 37-42.

Dell'Accio F, De Bari C, El Tawil NM, Barone F, Mitsiadis TA, O'Dowd J, Pitzalis C (2006) Activation of WNT and BMP signaling in adult human articular cartilage following mechanical injury. Arthritis Res Ther 8: R139.

Elders MJ (2000) The increasing impact of arthritis on public health. J Rheumatol Suppl 60: 6-8.

Fukui N, Zhu Y, Maloney WJ, Clohisy J, Sandell LJ (2003) Stimulation of BMP-2 expression by proinflammatory cytokines IL-1 and TNF-alpha in normal and osteoarthritic chondrocytes. J Bone Joint Surg Am 85-A Suppl 3: 59-66.

Gawri R, Antoniou J, Ouellet J, Awwad W, Steffen T, Roughley P, Haglund L, Mwale F (2013) Best paper NASS 2013: link-N can stimulate proteoglycan synthesis in the degenerated human intervertebral discs. Eur Cell Mater 26: 107-119. 
Gawri R, Ouellet J, Onnerfjord P, Alkhatib B, Steffen T, Heinegård D, Roughley $\mathrm{P}$, Antoniou J, Mwale F, Haglund L (2014) Link N is cleaved by human annulus fibrosus cells generating a fragment with retained biological activity. J Orthop Res 32: 1189-1197.

Goldring MB, Otero M (2011) Inflammation in osteoarthritis. Curr Opin Rheumatol 23: 471-478.

Hayashi M, Muneta T, Ju YJ, Mochizuki T, Sekiya I (2008) Weekly intra-articular injections of bone morphogenetic protein-7 inhibits osteoarthritis progression. Arthritis Res Ther 10: R118.

Henriksen M, Hunter DJ, Dam EB, Messier SP, Andriacchi TP, Lohmander LS, Aaboe J, Boesen M, Gudbergsen H, Bliddal H, Christensen R (2013) Is increased joint loading detrimental to obese patients with knee osteoarthritis? A secondary data analysis from a randomized trial. Osteoarthritis Cartilage 21: 1865-1875.

Hunter DJ (2011) Pharmacologic therapy for osteoarthritis - the era of disease modification. Nat Rev Rheumatol 7: 13-22.

Kluzek S, Sanchez-Santos MT, Leyland KM, Judge A, Spector TD, Hart D, Cooper C, Newton J, Arden NK (2016) Painful knee but not hand osteoarthritis is an independent predictor of mortality over 23 years follow-up of a population-based cohort of middleaged women. Ann Rheum Dis 75: 1749-1756.

Laverty S, Girard CA, Williams JM, Hunziker EB, Pritzker KP (2010) The OARSI histopathology initiative - recommendations for histological assessments of osteoarthritis in the rabbit. Osteoarthritis Cartilage 18 Suppl 3: S53-65.

Liu H, McKenna LA, Dean MF (1999) The macromolecular characteristics of cartilage proteoglycans do not change when synthesis is upregulated by link protein peptide. Biochim Biophys Acta 1428: 191-200.

Liu H, McKenna LA, Dean, MF (2000) An $\mathrm{N}$-terminal peptide from link protein can stimulate biosynthesis of collagen by human articular cartilage. Arch Biochem Biophys 378: 116-122.

McKenna LA, Liu H, Sansom PA, Dean MF (1998). An N-terminal peptide from link protein stimulates proteoglycan biosynthesis in human articular cartilage in vitro. Arthritis Rheum 41: 157-162.

Meachim G (1972) Light microscopy of Indian ink preparations of fibrillated cartilage. Ann Rheum Dis 31: 457-464.

Mwale F, Demers CN, Petit A, Roughley P, Poole AR, Steffen T, Aebi M, Antoniou J (2003) A synthetic peptide of link protein stimulates the biosynthesis of collagens II, IX and proteoglycan by cells of the intervertebral disc. J Cell Biochem 88: 1202-1213.

Mwale F, Masuda K, Pichika R, Epure LM, Yoshikawa T, Hemmad A, Roughley PJ, Antoniou J (2011) The efficacy of Link $\mathrm{N}$ as a mediator of repair in a rabbit model of intervertebral disc degeneration. Arthritis Res Ther 13: R120.

Mwale F, Masuda K, Grant MP, Epure LM, Kato K, Miyazaki S, Cheng K, Yamada J, Bae WC,
Muehleman C, Roughley PJ, Antoniou J (2018) Short Link $N$ promotes disc repair in a rabbit model of disc degeneration. Arthritis Res Ther 20: 201.

Noorwali H, Grant MP, Epure LM, Madiraju P, Sampen HJ, Antoniou J (2018) Link N as a therapeutic agent for discogenic pain. JOR Spine 1: e1008.

Poole AR, Kobayashi M, Yasuda T, Laverty S, Mwale F, Kojima T, Sakai T, Wahl C, El-Maadawy S, Webb G, Tchetina E, Wu W (2002) Type II collagen degradation and its regulation in articular cartilage in osteoarthritis. Ann Rheum Dis 61 Suppl 2: ii78-81.

Roughley PJ (2001) Articular cartilage and changes in arthritis: noncollagenous proteins and proteoglycans in the extracellular matrix of cartilage. Arthritis Res 3: 342-347.

Rountree RB, Schoor M, Chen H, Marks ME, Harley V, Mishina Y, Kingsley DM (2004) BMP receptor signaling is required for postnatal maintenance of articular cartilage. PLoS Biol 2: e355.

Salazar VS, Gamer LW, Rosen V (2016) BMP signalling in skeletal development, disease and repair. Nat Rev Endocrinol 12: 203-221.

Schmal H, Niemeyer P, Zwingmann J, Stoffel F, Sudkamp NP, Mehlhorn AT (2010) Association between expression of the bone morphogenetic proteins 2 and 7 in the repair of circumscribed cartilage lesions with clinical outcome. BMC Musculoskelet Disord 11: 170.

Sekiya I, Tang T, Hayashi M, Morito T, Ju YJ, Mochizuki T, Muneta T (2009) Periodic knee injections of BMP-7 delay cartilage degeneration induced by excessive running in rats. J Orthop Res 27: 1088-1092.

Spriet MP, Girard CA, Foster SF, Harasiewicz K, Holdsworth DW, Laverty S (2005) Validation of a $40 \mathrm{MHz}$ B-scan ultrasound biomicroscope for the evaluation of osteoarthritis lesions in an animal model. Osteoarthritis and cartilage 13: 171-179.

Stove J, Schneider-Wald B, Scharf HP, Schwarz ML (2006) Bone morphogenetic protein 7 (BMP7) stimulates proteoglycan synthesis in human osteoarthritic chondrocytes in vitro. Biomed Pharmacother 60: 639-643.

Tiraloche G, Girard C, Chouinard L, Sampalis J, Moquin, L, Ionescu M, Reiner A, Poole AR, Laverty $S$ (2005) Effect of oral glucosamine on cartilage degradation in a rabbit model of osteoarthritis. Arthritis Rheum 52: 1118-1128.

Wang Z, Hutton WC, Yoon ST (2013) ISSLS Prize Winner: The effect of link protein peptide (LPP) on human intervertebral disc cells. Spine (Phila Pa 1976) 38: 1501-1507.

Yoshioka M, Coutts RD, Amiel D, Hacker SA (1996) Characterization of a model of osteoarthritis in the rabbit knee. Osteoarthritis Cartilage 4: 87-98.

\section{Discussion with reviewer}

Laura Creemers: The injection procedures started 3 weeks after ACLT; OA likely was not developed yet. 
Authors: In prior animal model validation experiments in our laboratory we established that inflammation related to the surgical procedure persists up to 2 weeks, and established cartilage fibrillation and erosion is present as early as 4 weeks post-surgery (Spriet et al., 2005).

We elected to start therapy at 3 weeks and consider this as early post-traumatic OA. "fibrillation and erosion can occur as early as 4 weeks post ACL surgery" was indicated in the Discussion.

Laura Creemers: To what extent is this treatment prevention rather than actual treatment of OA?

Authors: Preventative therapy would have started immediately on day 1 post-operatively. Since fibrillation most likely already begun in the animal model following 3 weeks ACLT, this can be considered as a treatment.
Laura Creemers: What stage of human disease would this correspond to and would this be a stage patient would present themselves with at the clinician's?

Authors: We would consider this treatment as therapy for the early symptomatic stages of OA. The patients would likely present with slightly swollen and painful knees, with evidence of mild degenerative changes as noted on MRI or X-rays, but too early in the disease process to be considered surgical candidates. "treatment for early symptomatic stages of OA progression" was indicated in the Discussion.

Editor's note: The Scientific Editor responsible for this paper was Mauro Alini. 